\title{
Exploring XML Perturbation Techniques for Web Services Testing
}

\author{
Paulo Silveira and Ana C. V. de Melo \\ University of São Paulo \\ Department of Computer Science \\ São Paulo, Brazil \\ $\{$ silveira, acvm\}@ime.usp.br
}

\begin{abstract}
This paper presents testing techniques to automatically generate a set of test cases and data for web services. These techniques extend the ones based on Data Perturbation presented by Offutt and $\mathrm{Xu}$, to which are added mutation operators, boundary values considering XML Schema facets, testing cases using relationship defined in the message schema, UDDI integration and an internal database to collect and use values previously captured from messages. Together with these techniques, a tool (GenAutoWS ) was developed for proof of concepts.
\end{abstract}

\section{Introduction}

Most organizations today rely on information systems as part of their business process. The need to exchange data between different applications requires these applications to be more flexible and interoperable. Web Services emerged to support such requirements: services can communicate with each other by passing data from one service to another or by coordinating an activity between two or more services. Web services and SOA (Service-Oriented Architecture), which has been strongly implemented with Web services, have then received substantial attention from academy and industry.

The massive use of SOA and Web services in heterogeneous systems requires a high quality of development standards. Automated test tools, in particular, can help improving such quality. However, systems built on the top of Web services differ from traditional systems and testing techniques and tools applied to the latter cannot be directly applied to former.

Due to the particular features and the widespread use of Web services in industrial software, testing Web services has recently received more attention [11. In [6], Huang et al. pointed out two major approaches to address the Web services testing problem: automatic testing and model checking. New techniques have been proposed undergoing these approaches since then.

Tsai et al. [1] proposed a hierarchical testing framework to generate test scenarios based on WSDL (Web Service Description Language) specifications, together with some WSDL improvements. Huang et al. 6] presented a model checking process for OWL-S (Web Ontology Language for Web Services) in which 
the model checker BLAST [2] is extended to cope with concurrency in OWL-S. Some OWL-S extensions were also proposed. Song et al. 10] described a testing framework, named Coyote, that consists of a test master and a test engine. The test master allows the tester specifying test scenarios and cases, performing a set of analysis such as services dependency, completeness and consistency. The test engine interacts with Web services providing traces information.

Regarding the test automation approach, Offutt and $\mathrm{Xu} 8$ presented a Web Services testing technique based on data perturbation. Existing XML (Extensible Markup Language) messages are modified based on message grammars rules and data perturbation on values and interactions. The set of these modified messages are then used as test suites. Concerned with the automation approach, the present work extends the data perturbation testing technique by Offutt and $\mathrm{Xu}$ by adding mutation operators, boundary values considering XML Schema facets, testing cases using relationship defined in the WSDL, UDDI (Universal Description, Discovery and Integration) integration, internal database to collect and use values previously captured from messages. As proof of concept, a tool was developed, GenAutoWS , embedding the previous and the new techniques presented here.

The forthcoming sections present: some fundamental concepts on Web services and SOA; an existing technique to test Web services; the new testing techniques based on data perturbation; some experimental results regarding the new techniques; and, finally, some concluding remarks on the presented techniques.

\section{Web Services and SOA Preliminaries}

SOA (Service-Oriented Architecture) is essentially an architectural style to allow a collection of loosely coupled software agents interacting with each other [5]. The most common way of implementing such an architecture is by the use of Web Services.

There are today many definitions for Web Services. According to W3C [15], Web Services are software systems designed to support machine-to-machine interaction over a network via well-defined interfaces. A Web service is specified in a standard way by a service descriptor using a service description language, WSDL (Web Service Description Language [14]), for example. Each service descriptor must contain all the information needed to make the service interaction possible, including message format, transportation protocol and binding information.

Web services can interact with other systems in the way described by the service descriptors, using SOAP (Simple Object Access Protocol) to receive and send information. SOAP exchanges XML-based messages over another application layer protocol, normally HTTP (Hypertext Transfer Protocol) or MIME (Multipurpose Internet Mail Extensions). Those messages can differ in style, the two most common messages types are RPC (Remote Procedure Call) and Document. The RPC messages wrap program methods into the message, allowing them to be remotely invoked. The body contains a single element and all parameters are sub-elements. By contrast, in the Document style, the message content 
is placed directly into the body element, making Document-based Web services loosely coupled and document driven. A simple example of a Web Service message, using Document style, for a Movie Rental Store is shown in Listing 1.1. In this example, the driving license identifies the customer and the message contains a list of movies, each one with an id, price and media type.

Listing 1.1. XML document - Movie Rental Store

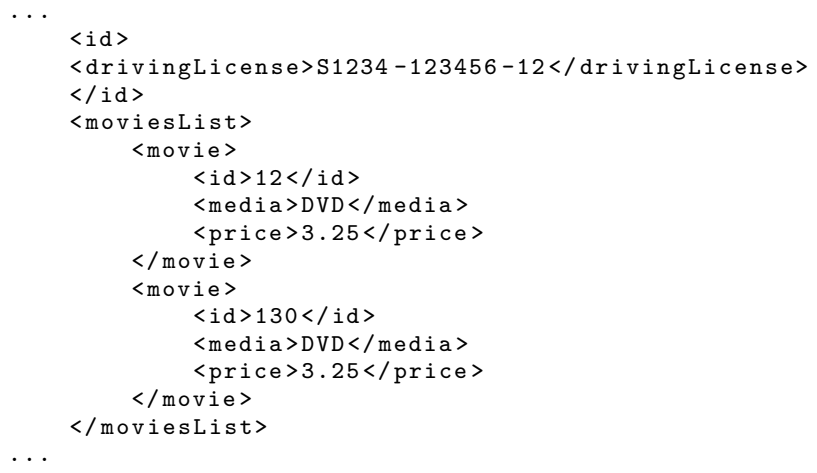

SOAP depends on XML standards, such as XML Schema and XML Namespaces. XML Schemas are used to describe messages exchanged by Web services. As such, Schemas define content, structure and semantics of XML documents that can be shared between applications. Restrictions on XML elements are called facets. Listing 1.2 shows an XML schema for the Listing 1.1. In this XML schema, we can see constraints on the elements: drivingLicense, media and price, it also uses the order indicator choice in the element ID and the occurrence indicator minOccurs and maxOccurs restricting the number of movies in this Web service call.

Listing 1.2. Schema for Movie Rental Store

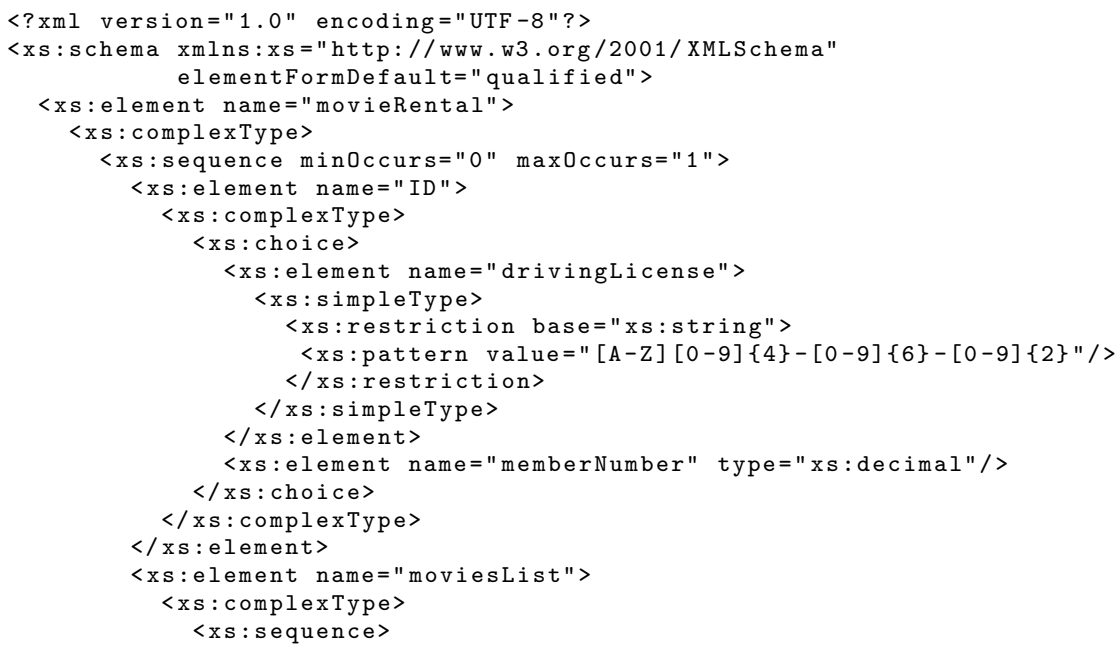


$\langle/$ xs: schema $>$

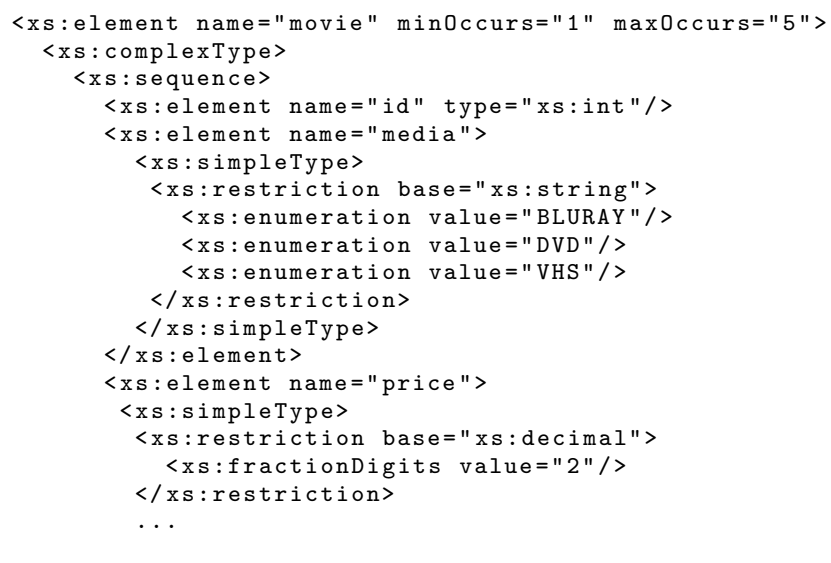

The Universal Description, Discovery and Integration (UDDI) 12 specification is used to catalog the Web Services. The implementation of this specification is called UDDI registry, representing data and metadata about Web Services. UDDI registry includes a set of Web Services to allow service to be published and found.

Web services are rather used in a very distributed and heterogenous contexts and require dynamic integration. Their applications interact in three different ways: publishing, the service provider makes a service interface available to other services; finding, other services (requesters) must be able to discover the service interface; binding, address the ability to connect and invoke services. Many of these features make Web services differ from traditional and web applications and the testing must be performed accordingly. Web services must be tested considering also communication aspects [8]1]: discovering Web services, the data format exchanged, and the request/response mechanisms. Testing SOAP messages addresses request/response mechanism and data format aspects of Web services. WSDL is used to expose interfaces as services available on the Internet. Testing WSDL files can be used to generate test plans to validate services. Testing UDDI registries provides the capabilities of publishing, finding and binding of SOA, giving the way software is integrated.

\section{A Web Services Testing Technique Based on Data Perturbation}

Data perturbation testing technique consists of changing (perturbing) existing data to create new test sets. For Web Services, Offutt and $\mathrm{Xu}$ [8] presented a data perturbation technique based on data value and interaction perturbations. Data value perturbation modifies values using data type information, following the boundary value testing approach [1. For this, a set of rules for XML data types, corresponding to the primitive types in most programming languages, were created. Table 1 shows the data types with the corresponding 
Table 1. Data value perturbation

\begin{tabular}{|l|l|}
\hline Data Type & Boundary Values \\
\hline String & Maximum length, minimum length, upper case, lower case \\
\hline Numeric & Maximum value, minimum value, zero \\
\hline Boolean & true, false \\
\hline
\end{tabular}

data value perturbations to be applied. Then, for each test data, new ones are created based on the boundary values.

For interaction perturbation, messages are modified according to their types: $R P C$ or data communications. For RPC messages, testing is focused on data uses and mutation operators. The traditional mutation operators were redefined for Web Services [8, as shown in Table 2

Table 2. Data type operators

\begin{tabular}{|l|l|}
\hline Divide $(n)$ & Change value $n$ to $1 \div n$, where $n$ is double data type \\
\hline Multiply $(n)$ & Change value $n$ to $n \times n$ \\
\hline Negative $(n)$ & Change value $n$ to $-n$ \\
\hline Absolute $(n)$ & Change value $\mathrm{n}$ to $\mathrm{n}$ \\
\hline Exchange $\left(n_{1}, n_{2}\right)$ & Substitute value $n_{1}$ for $n_{2}$ and vice-versa, where $n_{1}$ and $n_{2}$ have the same type. \\
\hline Unauthorized $(s t r)$ & Change string value $s t r$ to str' $O R$ ' 1 ' $=$ '1 \\
\hline
\end{tabular}

For data communications (document-based messages), testing focus on relationships and constraints (defined by facets in XML Schemas). To precisely define these, XML Schema are defined using RTG (Regular Tree Grammar), a formal model for XML schemas.

Definition 1. A regular tree grammar is a 6 -tuple $<E, D, N, A, P, n_{s}>$, where:

1. E is a finite set of element types

2. $D$ is a finite set of data types

3. $N$ is a finite set of non-terminals

4. A is a finite set of attribute types

5. $P$ is a finite set of production rules with two forms:

- $n \rightarrow a\langle d\rangle$, where $n$ is non-terminal in $N$; a is either an attribute type in $A$ or an element type in $E$, and $d$ is a data type in $D$;

$-n \rightarrow e<r>$, where $n$ is non-terminal in $N$; $e$ is an element in $E$, and $r$ is a regular expression comprised of non-terminals.

6. $n_{s}$ is the starting non-terminal, $n_{s} \in N$

Based on the maxOccurs attribute of XML schemas (a relationship defined as XML facet - see Listing 1.2, for example), the parent-child associations are acquired and a regular expression for the relationship is created 1 . For these relationships, some testing strategies were defined[8]:

${ }^{1}$ In the regular expressions, operators '?', '十', and '*', denote zero-or-one, at least one, and any number of element occurrences, respectively. These operators reflect cardinality constraints in an XML Schema. 
- Given a relationship $n \rightarrow e<r>$, if there is an expression $\alpha$ ? in $r$, there will be two test cases: an $\alpha$ and an empty instance.

- Given a relationship $n \rightarrow e<r>$, if there is an expression $\alpha+$ in $r$, there will be two test cases: an $\alpha$ and a number of $\alpha$ instances.

- Given a relationship $n \rightarrow e<r>$, if there is an expression $\alpha^{*} \alpha$ in $\mathrm{r}$, there will be two test cases. One contains $\alpha^{*} \alpha$ and the other contains $\alpha^{*-1}$, where $\alpha^{*} \alpha$ duplicates one element instance and $\alpha^{*-1}$ deletes one element instance.

Besides that strategy based on relationships, in [16], a method to generate tests for XML-based communication by modification and further instantiation of XML schemas was presented. Schemas are modified based on predefined perturbation operators. The goal is to perturb XML Schemas to create invalid XML messages. With this aim, seven perturbation operators for XML Schema were defined; some are applied to nodes and others to sub-trees. For nodes, the operators are: insert and delete a new node between two other nodes, insert and delete a new node with a data type under an existing node. The sub-tree operators are: insert and delete a sub-tree below a node and change an edge between two nodes using an edge with different constraints. Almeida and Vergilio 4 extended that work with six new mutation operators for SOAP messages, together with a tool to generate modified messages. Table 3 shows the operators defined by them.

Table 3. Mutation operators - SOAP

\begin{tabular}{|l|l|}
\hline Operator name & Brief description \\
\hline Null $(n)$ & Set to null the value assigned to a node $n$ in the SOAP message. \\
\hline Incomplete $(n)$ & Delete a node $n$ and its child nodes from the SOAP message. \\
\hline Inversion $(n)$ & Inverts the order of nodes within node $n$ in the given SOAP message. \\
\hline ValueInversion $(n)$ & $\begin{array}{l}\text { Inverts the order of the values assigned to the child nodes of node } n \text { in } \\
\text { the given XML message. }\end{array}$ \\
\hline Mod_Len $(n)$ & $\begin{array}{l}\text { Modifies the length of the value assigned to node } n \text { in the given XML } \\
\text { message. }\end{array}$ \\
\hline Space $(n)$ & Set to ' the value assigned to node $n$. \\
\hline
\end{tabular}

\section{A New Technique on Test Cases Generation for Web Services}

This section presents a new testing technique based on [8] and [4]. The extensions to the previous works aim to increase the test coverage, creating new messages with information not explored by original works, mainly from XML Schemas:

1. boundary analysis is enlarged with values immediately above and below the data type domain, as defined by Pressman [9] and Myers 7];

2. XML facets are also considered in boundary analysis;

3. new relationship rules are added to data communication perturbation, including choice and all, and the occurrence indicator minOccurs; and

4. four new mutation operators are defined for RPC messages.

Either for data value and interaction perturbation, invalid cases are also considered. This means that the Web Service should return an error when test suites 
corresponding to these test cases are executed. For example, if the valid set of values for a given element is 1,2 and 3 and the message generated has the value 4 , this test case will be classified as invalid. This property intends to help in the oracle test.

\subsection{Extending Data Value Perturbation}

Web services using literal messages can be defined by XML Schema and the legal values for each simple type can be constrained using XML Schema Facets. There are twelve different Facets. Offutt and $\mathrm{Xu}$ [8 has considered only the maximum and minimum values and the totaldigits Facets under valid values. We improved the test of these facets by adding test cases for invalid values (when executed, these messages should receive an error message). Apart from these, all other XML Schema Facets were considered to create new messages. We use these Facets to assist in the data value perturbation. Here, the facets and the corresponding test cases are presented.

Pattern: Defines the valid content for a data type, specified by a regular expression. We use pattern values to generated valid and invalid messages. For the drivingLicense type definition showed in Listing 1.2, the new messages in Table 4 corresponds to the test suites generated for the Pattern test cases:

Table 4. Test suites for pattern in Listing 1.2

\begin{tabular}{|l|l|}
\hline$<$ drivingLicense $>$ Z9999-999999-99 $</$ drivingLicense $>$ & Valid \\
\hline$<$ drivingLicense $>$ A0000-000000-00 $</$ drivingLicense $>$ & Valid \\
\hline$<$ drivingLicense $>$ 9ZZZZ-ZZZZZZ-ZZ $</$ drivingLicense $>$ & Invalid \\
\hline
\end{tabular}

A regular expression quantifier specifies how often that preceding character or group is allowed to occur. A predefined number should be used to create test cases to messages that has the quantifier ' $*$ ' or ' + '.

Enumeration: Constrains the valid values of a data type to a specified set. A message is generated for each value in the given enumeration set. An invalid message is also generated with a value out of this set. For the media type definition presented in Listing [1.2, the technique generates the messages showed in Table 5

Table 5. Test Suites for enumeration

\begin{tabular}{|l|l|}
\hline$<$ media $>$ DVD $</$ media $>$ & Valid \\
\hline$<$ media $>$ VHS $</$ media $>$ & Valid \\
\hline$<$ media $>$ ZZZZZZZZ $</$ media $>$ & Invalid \\
\hline
\end{tabular}

FractionDigits: Specifies the maximum number of digits allowed in the fractional part of numbers. The value must be equal or greater than zero. Three messages are generated: one with the maximum number of digits, the second with one digit and an invalid message with oversized fractional digits. 
Length: Specifies the number of character or list items are allowed. A valid message and an invalid message are generated. The invalid message has an extra character than the maximum allowed.

TotalDigits: Defines the maximum number of values are allowed by restricting it to numbers expressible as $i \times 10^{-n}$, where $i$ and $n$ are integers such that $|i|<10^{\text {totalDigits }}$ and $0 \leq n \leq$ totalDigits. Example: using totalDigits $=4$, the value of 55.51 is valid, it can be expressed as $5551 \times 10^{-2}, i=5551$ and $n=2$. A valid message is generated using the maximum number of digits allowed and an invalid message is created using a value over this maximum value. An extra message is generated with fractional digits if the facet FractionDigits is also specified for this element.

WhiteSpace: Specifies how spaces, line feeds, tabs, and carriage returns will be handled. Depending on whitespace value (preserve, replace, collapse) messages are generated including line feeds, tabs and carriage returns.

For all other data type facets (maxInclusive, minInclusive, maxExclusive, minExclusive, maxLength, minLength), data value perturbation defined by Offutt and $\mathrm{Xu}$ is applied to generate the test cases.

Test cases generated using XML Schema facets will test boundaries values not only based in the data type but also in constrains defined for the element. The more accurate an element is specified, the more effective is the quality of the messages generated. Also, the extensions for data value perturbation based on values immediately above and below the data type domain tests messages for invalid domains.

\subsection{Extending Relationship Strategies for Data Communication Perturbation}

In Document-based Web services, service consumer and provider interact using complete documents. These documents are typically XML files, defined in a common way, agreed upon schema. Data communications aim at testing Documentbased Web service. DCP (Data Communication Perturbation) focus on testing relationship and constraints in this kind of messages. As with 8, here messages are defined using RTG (Regular Tree Grammar) - Definition 1, and the relationships and constraints are the finite set of production rules $P$ in the RTG.

Definition 2. Given an XML schema $<E, D, N, A, P, n_{s}>$, a relationship is a production rule in $P: n \rightarrow e<r\rangle$, where $n$ is a non-terminal in $N, e$ is an element in $E$, and $r$ is a regular expression made up of non-terminals.

Offutt and $\mathrm{Xu}$ used the occurrence indicator maxOccurs to specify referential relationships between parent and child elements. Here, this idea is extended with the use of the occurrence indicator minOccurs, and the order indicators: all and choice, and the element any. Table 6] describes each of the XML Schema indicator used and the corresponding regular expression. 
Table 6. Regular expressions used to represent relationship in the RTG representation

\begin{tabular}{|l|l|l|}
\hline XML Schema indicator & regexp & description \\
\hline minOccurs, maxOccurs & $\{x, y\}$ & at least $x$ and not more than $y$ times \\
\hline choice & & one child element or another can occur \\
\hline all & $\left\{x_{1}, . ., x_{n}\right\}$ & $\begin{array}{l}\text { the child elements can appear in any order but each must } \\
\text { occur only once. }\end{array}$ \\
\hline any element &. & element not specified in the XML Schema \\
\hline
\end{tabular}

Apart from the three testing strategies established by Offutt and Xu (Section 3), the following testing strategies are added:

- Given a relationship $n \rightarrow e<r>$, if there is an expression $\alpha+$ in $r$, there will be one extra test case that contains no instances of $\alpha$. This test case expect to receive an error when executed.

- Given a relationship $n \rightarrow e\langle r\rangle$, if there is an expression $\alpha *$ in $r$, there will be two extra test cases. One deleting all instances of $\alpha$, and the other one containing $k$ instances of $\alpha$, where $k$ is a predefined number representing unbounded.

- Given a relationship $n \rightarrow e<r>$, if there is an expression containing '.' in $r$, there will be one test case. It contains one instance of $\beta$, where $\beta$ represents any element.

- Given a relationship $n \rightarrow e<r>$, if there is an expression $\alpha\{x, y\}$ in $r$, there will be two test cases. One contains $x$ instances of $\alpha$ and the other contains $y$ instances of $\alpha$. If $y$ has the value unbounded, $y$ will have the value of $k$, where $k$ is a predefined number.

- Given a relationship $n \rightarrow e<r>$, if there is an expression $\left\{x_{1}, . ., x_{n}\right\}$ in $r$, there will be two test cases. One contains a random permutation of $\left\{x_{1}, . ., x_{n}\right\}$, and the other one contains $\left\{x_{1}, . ., x_{n-1}\right\}$.

- Given a relationship $n \rightarrow e<r>$, if there is an expression $x_{1}|..| x_{n}$ in $r$, there will be $n+1$ different test cases. The first $n$ tests case contains $x_{i}$ where $i$ is an integer and $1 \leq i \leq n$. The other test cases will contains all $n$ elements, this last expects to receive an error when executed.

The RTG for the XML Schema showed in the Listing 1.2 contains two relationships:

$n_{\text {id }} \rightarrow I D<n_{\text {drivingLicense }} \mid n_{\text {memberN }}$ umber $>$

$n_{\text {movielist }} \rightarrow$ movie $<\left(n_{\text {movieId }}, n_{\text {media }}, n_{\text {price }}\right)\{1,5\}>$

Four test cases for both relationships are shown in Listings 1.3, 1.4, 1.5, 1.6.

Listing 1.3. Test data for choice -2nd element

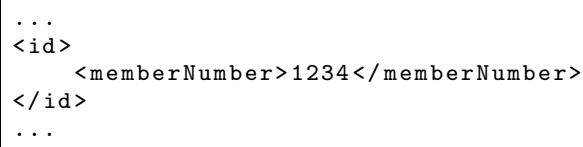


Listing 1.4. Test data for all choice elements- An error is expected for this message

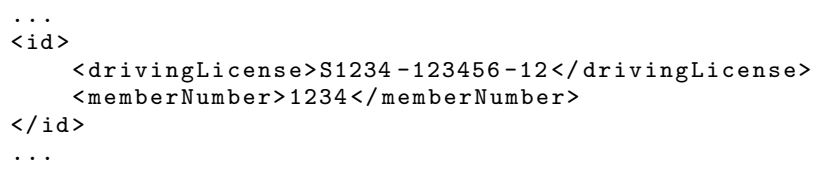

Listing 1.5. Test suites for the maximum number allowed for sequence's relationship

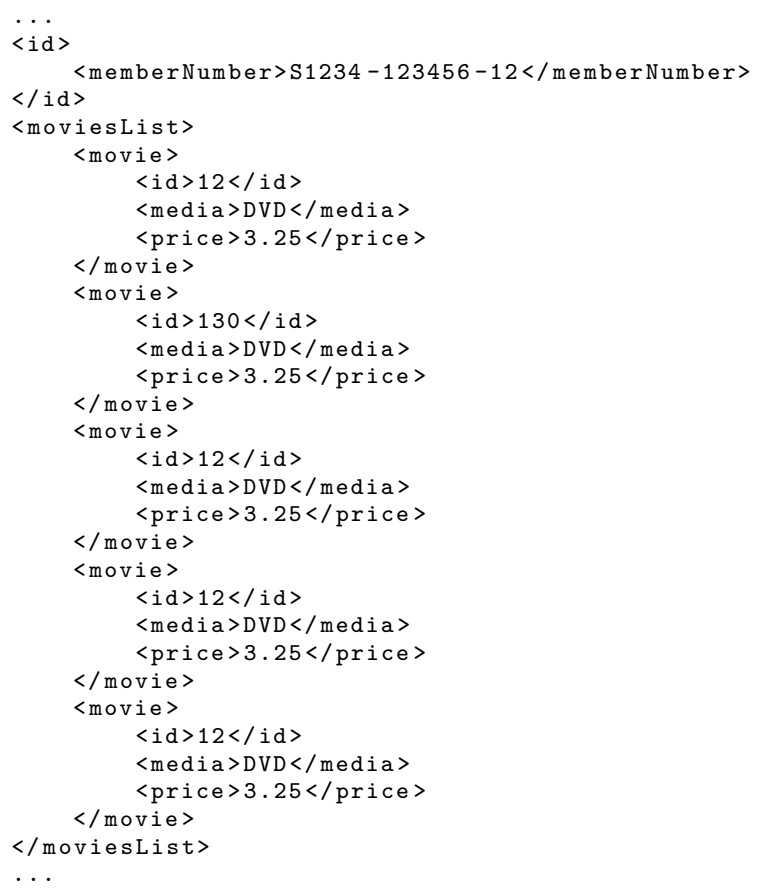

Listing 1.6. Test suites for the minimum number allowed for sequence's relationship

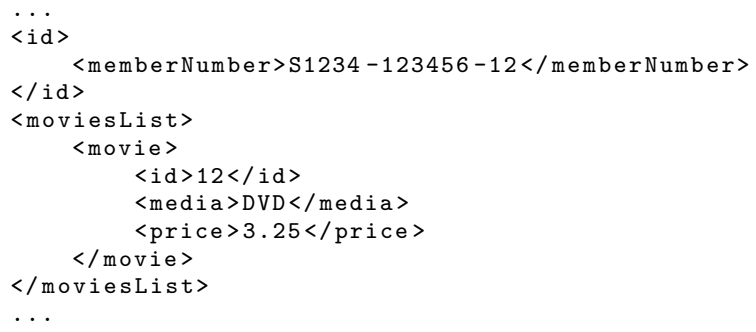

\subsection{Operator Perturbation}

Operator Perturbation relies on the idea of RPC Communication Perturbation 8 and SOAP Perturbation Operators [4]. In [8], mutation operators were only 
applied to RPC style Web services. In the present work, all mutation operators, presented in Table 2] and 3, are implemented for RPC Web services and, besides that, all of them are redefined and implemented for Document based Web services. Apart from those already defined operators, the following new mutation operators are defined:

Code Injection: Code injection is a technique to introduce some code into a program. The goal normally is to take advantage of some assumption, such as: quotation marks or semi-colons would never appear, only alphanumeric will be entered, use of array index from input, and so on. The following mutation operator were included:

DynamicEvaluation $(\boldsymbol{n})$ - This mutation operator explores the situation when part of input is used into an eval function, Listing [1.7 shows an example of code.

Listing 1.7. Code using function eval

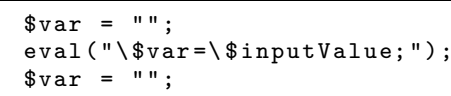

Test case: Add the value: 0 ; system $\backslash$ "/bin/echo error message $\backslash ") ;$ to the node $n$.

FileInjection $(\boldsymbol{n})$ - This operator is used to demonstrate errors when an input parameter is used as file name.

Test case: Add a file path as element value.

Numeric Value SQL Injection: To protect against SQL injection, many programmers are escaping or removing quotes, but this does not completely remove the risk in certain programming languages. Consider the following query:

SELECT fields FROM table WHERE id == $\$$ id;

The variable $\$$ id was assumed to be a numeric value and it would expose all users if someone tries: $i d=0$ OR $1=1$.

Test case: Add the value $=0$ OR $1=1$ to the element value.

Null: XML Schema introduces a mechanism for signaling that an element's content is missing or "null" 13. This operator only applies for literal messages. Listing 1.8 has an extract of XML Schema that specify nullable and a XML that has a null element.

Test Case: Remove the element value and add the attribute xsi:null="true" to the element.

Listing 1.8. Null example

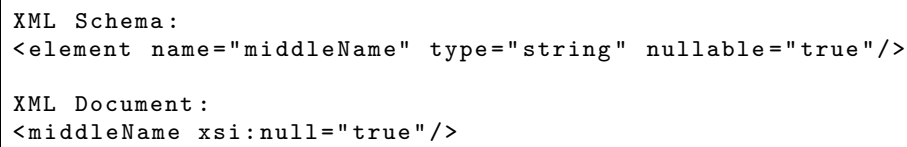


The following mutation operator was included:

\section{$\operatorname{Null}(\mathbf{n})$}

Test case: Add the attribute xsi:null="true" to the node $n$, and delete its contents.

\section{Empirical Results}

Using the testing techniques presented in this paper, we built a tool, GenAutoWS. It can be used by both Web services subscribers and providers. For the former, the test suites are generated based on the service interfaces to certify particular uses of services. Service providers can also be benefited by the use of GenAutoWS as a development tool in which messages are automatically generated based on the presented techniques.

Test cases automatically generated by the tool can be included in a test suite. These test suites can actually be executed by the tool which is responsible for sending messages to the Web services and response messages can be checked by the tester. Messages perturbed to create new test cases are automatically saved in an internal database. GenAutoWS has a feature, namely "internal data perturbation", used to create new messages by swapping values with previously saved values.

Regarding connectivity GenAutoWS supports UDDI. Bloomberg [3] showed many issues of testing Web services including testing publishing, finding and binding capabilities of SOA. GenAutoWS allows UDDI registry inquiries, the WSDL returned is used to create test case messages automatically. The seed message uses values from the internal database or values default based on the element datatype.

GenAutoWS was submitted as a first proof-of-concept to generate test cases for five Web services from two different systems of a financial institution. The first three Web services (WS1, WS2 and W3) belong to an enterprise email application and the other two Web services (WS4 and WS5) are used to verify credit information.

The Web services used in this proof-of-concepts are specified using WSDL and they communicate using SOAP over HTTP. The first four Web services are document/literal and WS5 is RPC/encoded. Both systems were developed in Java. Although they are now used in a production environment, pre-release versions of these systems were used for test. During the tests, the faults found were classified as low, medium and high accordingly to the critical level.

Using the techniques of boundary value [8] and all XML Schema Facets presented in Section 4.1, we generated 162 different tests. Table 7 summarizes the result for this approach. The majority of the observed faults were classified as low level, example: no error message or message incomplete.

The relationship strategies for DCP, shown in Section 4.2, generated 49 tests, the results are presented in Table 8 . No tests were generated for WS5 using this technique, since it was not specified by XML Schema.

\footnotetext{
${ }^{1}$ Some faults were found for more than one test cases.
} 
Table 7. Tests - Data Value Perturbation

\begin{tabular}{|l|r|}
\hline Number of tests & 162 \\
Generated using the new techniques & 99 \\
Generated using the previous techniques & 63 \\
\hline Total number of faults & 32 \\
Medium and high level faults & 7 \\
Tests that detect faults 1 & 62 \\
From new techniques & 48 \\
From previous techniques & 14 \\
\hline
\end{tabular}

Table 8. Tests - Data Communic. Perturbation

\begin{tabular}{|l|r|}
\hline Number of tests & 49 \\
$\quad$ Generated using the new techniques & 40 \\
$\quad$ Generated using the previous techniques & 9 \\
\hline Total number of faults & 12 \\
Medium and high level faults & 4 \\
Tests that detect faults ${ }^{2}$ & 20 \\
$\quad$ From new techniques & 16 \\
From previous techniques & 4 \\
\hline
\end{tabular}

Table 9. Tests - Mutation Operators

\begin{tabular}{|l|r|}
\hline Number of tests & 164 \\
$\quad$ Generated using the new techniques & 48 \\
$\quad$ Generated using the previous techniques & 116 \\
\hline Total number of faults & 16 \\
Medium and high level faults & 4 \\
Tests that detect faults 1 & 44 \\
$\quad$ From new techniques & 10 \\
From previous techniques & 34 \\
\hline
\end{tabular}

The mutation operators presented in 84 and the four new operators proposed in Section 4.3 generated 164 tests. The results are summarized in the Table 9.

Certain faults were detected by multiple tests and were common among the different techniques. The new techniques presented in the present paper generated more test cases/data than the original approach for the DVP and DCP tests. These new tests could reveal faults not detectable by the original techniques. The new testing strategies defined for DCP took advantage of relationships described in XML Schema not considered before. For instance, the order indicators choice and $a l l$. The generation of test cases/data that should cause errors in the application allowed us to validate errors messages returned by the service, either for incorrect or inexistent messages.

The use of other XML Schema constrains (facets) in the DVP technique permitted to create more accurate data within the element domain. For example, for the string element that contain pattern: $[\mathrm{A}-\mathrm{Z}]\{2,3\}-[0-9]\{2,3\}$, a part from the string maximum length from the previous techniques, two new ones were created: ZZZ-999 and ZZZZ-9999. The latter is an invalid message and should receive an error when executed. 


\section{Conclusion}

This paper proposed extensions to testing techniques based on Data Perturbation for Web Services together with a tool to generate the test suites based on the previous and new techniques. For that, new mutation operators, boundary values considering all WSDL facets, testing cases using relationship defined in the WSDL, UDDI integration, internal database to collect and use values previously captured from messages were proposed.

The testing tool GenAutoWS was built using these extensions and a first proof-of-concepts using five Web services from a financial institution was carried out. In this proof-of-concepts, DVP tests were shown more efficient compared to the other techniques, founding at least twice the number of faults. Such a result is similar to the one got by Offutt and $\mathrm{Xu}$ [8] in their proof-of-concepts.

The test cases added to DVP and DCP previous techniques could generate more messages and reveal more faults than their counterparts in the original approach. The new rules inserted to DCP and the improvements for DCP were able to generate many messages for WS1, WS2 and WS3. These Web Services had many constrains specified using XML Schema facets and XML Schema order indicators testing technique could exploit these features. Although the mutation operators presented in the section 4.3 have a limited scope in the case studies preformed so far, they can produce better results if applied to systems in which security issues are more relevant.

Web Services testing presented here can be applied to existing Web Services without modifying or rewriting any piece of code, or adopting a specific framework. The quality of test cases/data generated, however, depends on how well the Web Services are specified, since the WSDL and schema files are used to generate the messages.

The techniques for data perturbation based on rules for XML Schema can be easily adapted to different kinds of applications that exchange messages in XML format. One possible work is to explore the generation of test cases for REST (Representational State Transfer) with information specified in a WADL (Web Application Description Language) file, a message descriptor for REST Services, using the same techniques presented here.

Acknowledgements. This project has been co-funded by the National Council for Scientific and Technological Development (CNPq - Brazil) - Process:551038/20071, the State of São Paulo Research Foundation (FAPESP) and the Ministry of Education Research Agency (CAPES- Brazil) - Process:0671-08-8.

\section{References}

1. Beizer, B.: Software Testing Techniques, 2nd edn. International Thomson Computer Press (1990)

2. UC Berkeley. Blast (berkeley lazy abstraction software verification tool) model checker, http://embedded.eecs.berkeley.edu/blast/ 
3. Bloomberg, J.: Testing web services today and tomorrow. The Rational Edge Ezine for the Rational Community (2002)

4. de Almeida Jr., L.F., Vergilio, S.R.: Exploring perturbation based testing for web services. In: ICWS 2006: Proceedings of the IEEE International Conference on Web Services (ICWS 2006), Washington, DC, USA, pp. 717-726. IEEE Computer Society, Los Alamitos (2006)

5. Harrison, A., Taylor, I.J.: Wspeer - an interface to web service hosting and invocation. In: IPDPS 2005: Proceedings of the 19th IEEE International Parallel and Distributed Processing Symposium (IPDPS 2005) - Workshop 4, Washington, DC, USA, p. 175. IEEE Computer Society, Los Alamitos (2005)

6. Huang, H., Tsai, W., Paul, R., Chen, Y.: Automated model checking and testing for composite web services. In: ISORC 2005: Proceedings of the Eighth IEEE International Symposium on Object-Oriented Real-Time Distributed Computing (ISORC 2005), Washington, DC, USA, pp. 300-307. IEEE Computer Society, Los Alamitos (2005)

7. Myers, G.J.: The Art of Software Testing, 2nd edn. Wiley, New York (2004)

8. Offutt, J., Xu, W.: Generating test cases for web services using data perturbation. SIGSOFT Softw. Eng. Notes 29(5), 1-10 (2004)

9. Pressman, S.R.: Software Engineering: A Practitioner's Approach, 6th edn. McGraw-Hill, New York (2004)

10. Tsai, W.T., Paul, R., Song, W., Cao, Z.: Coyote: An xml-based framework for web services testing (2002)

11. Tsai, W.T., Paul, R., Yu, L., Saimi, A., Cao, Z.: Scenario-based web service testing with distributed agents (2003)

12. OASIS UDDI. UDDI Specification, http://www.uddi.org/specification.html (last access, 2005)

13. W3C. Xml schema part 1: Structures, http://www.w3.org/TR/2000/CR-xmlschema-1-20001024/

14. W3C. Web services description language (WSDL) version 2 part 1: Core language, http://www.w3.org/TR/wsdl20/ (last access, 2006)

15. W3C. Web services glossary (last access, July 2007)

16. Xu, W., Offutt, J., Luo, J.: Testing web services by xml perturbation. In: ISSRE 2005: Proceedings of the 16th IEEE International Symposium on Software Reliability Engineering, Washington, DC, USA, pp. 257-266. IEEE Computer Society, Los Alamitos (2005) 